\title{
Present and future of hadron spectroscopy at Jefferson Lab
}

\author{
M.Battaglieri (for the CLAS Collaboration) \\ Istituto Nazionale di Fisica Nucleare, Genova, Italy
}

\begin{abstract}
The CLAS Collaboration is operating the CLAS detector at theThomas Jefferson National Laboratory (JLab) in USA. The unique combination of the detector large acceptance and high intensity of the continuous electron beam of CEBAF has opened the way to a comprehensive study of the hadrons structure in kinematic domain between nuclear and particle physics. Hadron spectroscopy plays a central role in the physics program of the Collaboration. Many exclusive channels have been studied with virtual and real photon beams in a wide kinematic providing key information about the hadron structure as well as the reactions dynamic. In this contribution, the rich physics program covered by present and future experiments will be reviewed.
\end{abstract}

\section{Introduction}

The use of electromagnetic probes in hadron physics has a long history and led to important discoveries: the proton electric and magnetic form factors, the scaling law of deep inelastic scattering etc. Many other experiments were performed thereafter, aiming at the study of particular exclusive channels like pion photo- and electro-production, but mostly with limited statistics and accuracy, due to the low luminosity and duty factors of the machines previously available, as well as to the small detector solid angles. The availability of continuous and high intensity electron and photon beam together with a new large acceptance detector provides coverage over a wide kinematic range for many different final states, including multi-hadron channels. Hadron spectroscopy, and in particular the study of meson spectrum and reactions dynamic, is one of the most effective tool to have a quantitative understanding of quark and gluon confinement. In fact, revealing the nature of the mass of the hadrons as well as seeing the QCD degrees of freedom (e.g. gluons) at work is one of the remaining issue addressed by many current experiments and it is at the top of the physics program of future facilities.

The CLAS collaboration in Hall B at Jefferson Laboratory has a broad scientific program based on the use of both the primary $\mathrm{CW}$ electron beam and a tagged photon beam. In our kinematics (center of mass energy $W \sim 1-3 \mathrm{GeV}$, photon virtuality $Q^{2} \sim 0-5 \mathrm{GeV}^{2}$, and momentum transfer in exclusive reactions $-t \sim 0-6 \mathrm{GeV}^{2}$ ) it is possible to study the transition between the so called 'Soft Physics', where models based on Constituent Quarks or phenomenology are widely applied, to the 'Hard Physics' regime, where perturbative QCD or dimensional analysis are used to interpret the data. In this contribution I will show some selected results obtained by the CLAS Collaboration in baryon and meson spectroscopy as well as the hadron spectroscopy program at CLAS12.

\section{CLAS at Jefferson Lab}

The Continuous Electron Beam Accelerator Facility (CEBAF) at Jefferson Lab delivers a low emittance, high resolution, $100 \%$ duty-cycle electron beam to three different experimental halls A, B and C, simultaneously. The maximum energy is $5.8 \mathrm{GeV}$ (with $80 \%$ polarization available) with a maximum current of $180 \mu \mathrm{A}$. The Hall B is mainly devoted to experiments that require the detection of several particles in the final state. It hosts the CLAS detector [1] (CEBAF Large Acceptance Spectrometer), built around six superconducting coils producing a toroidal magnetic field. The detector package consists of three layers of drift chambers for track reconstruction, one layer of scintillators for time-of-flight measurements and hadron identification, forward Cerenkov counters for electron-pion discrimination, and electromagnetic calorimeters to identify electrons and neutral particles. The detector lay-out is sketched in Fig. 1. 


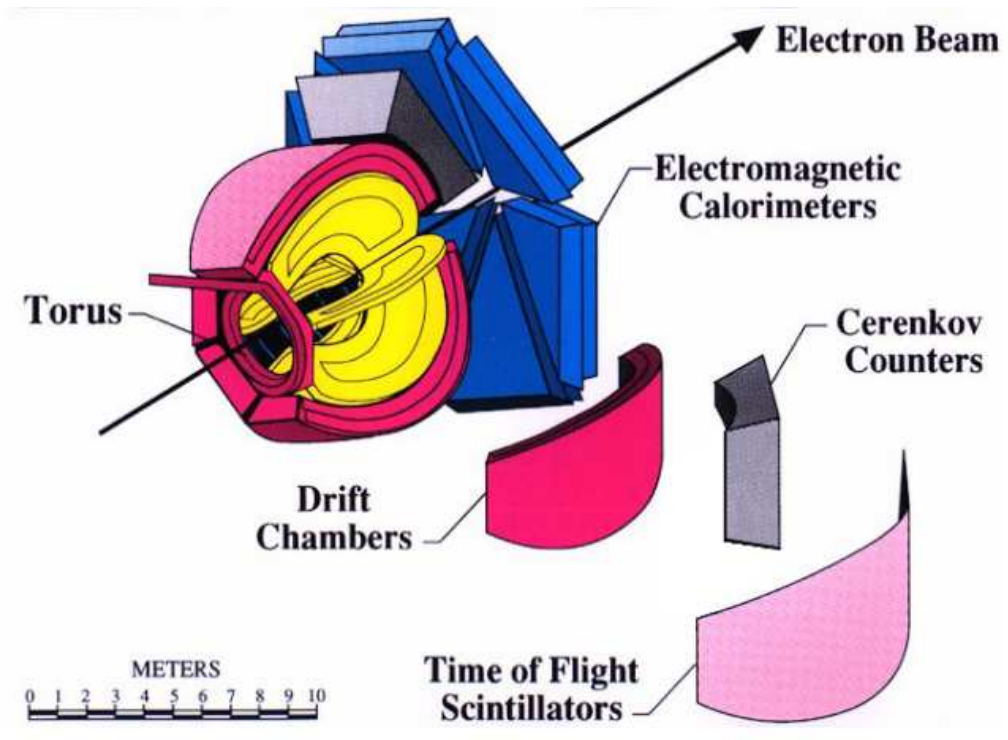

Fig. 1: The components of the CLAS detector.

\section{Baryon spectroscopy: the $N^{*}$ program}

One of the features of real and virtual photon interactions with the proton is the appearance of bumps in the inelastic part of the cross section, corresponding to values of the invariant $\mathrm{CM}$ energy $W$ between 1.1 and about $2 \mathrm{GeV}$. This is interpreted as manifestation of excited states of the nucleon (with definite angular momentum and parity), also observed in pion absorption and nucleon-nucleon scattering. Despite the progress being made within the framework of quark models to explain the resonances in terms of three quarks, the number of states observed in the spectrum is higher than what predicted by any symmetric quark model based on SU(6). A possible explanation concerns the underlying symmetry: e.g. the Quark Cluster Model, where two of the three constituents stay together in a di-quark configuration leading to a fewer number of predicted states. Another possibility is related to the QCD mixing effects that could decouple many of these states from the pion-nucleon channel, with a consequent lack of evidence in elastic $\pi N$ scattering, while strongly coupling them to multipion channels $(N \pi \pi)$ or higher masses mesons $(N \eta, N \omega, k Y)$. Search for the states still missing in the experimental observation is therefore crucial in understanding the basic degrees of freedom in baryon structure. As an example, I briefly report the CLAS results for the exclusive reaction $e p \rightarrow e^{\prime} p \pi^{+} \pi^{-}$[2]. The new CLAS data show for the first time a clear evidence of the presence of bumps in the total cross section at different $Q^{2}$ values, and a 'missing' strength around $W \sim 1.7 \mathrm{GeV}$ (see fig. 2). The comparison to a phenomenological model incorporating the available knowledge on $N^{*}$ and $\Delta$ states shows an evident discrepancy. A better description of the data is obtained either by a sizable change of the $P_{13}(1720)$ resonance or by introducing a new baryon state not reported in published analysis.

Indications of different contributions to baryon dynamics, beside the standard $q q q$ configurations, comes from the study of transition from the ground to the first excited state $(N \rightarrow \Delta(1232))$. Within the SU(6) model, the $\gamma^{*} N \rightarrow \Delta(1232) \rightarrow N \pi$ transition is mediated by a single quark spin flip leading to $M_{1+}$ magnetic dipole dominance and $E_{1+}=S_{1+}=0$. Non-zero values for $E_{1+}$ would indicate a quadrupole deformation. Such deformation may arise dynamically through the interaction of the photon with the pion cloud or through the one-gluon exchange mechanism. On the other hand, helicity conservation in perturbative QCD requires $E_{1+}=M_{1+}$ as $Q^{2} \rightarrow \infty$. Precise determination of the ratio $\frac{E_{1+}}{M_{1+}}$ is therefore an important test about the underlying quark structure of the nucleon. Results of the multipole analysis of the CLAS data [3] for the reaction $e p \rightarrow e^{\prime} p \pi^{0}$ are shown in figure 3. These data, extended to $Q^{2}$ up to $6 \mathrm{GeV}^{2} / \mathrm{c}^{2}$ by a recent analysis prove that $R_{E M}$ remains negative and small in the whole kinematic range of our measurement, indicating that $\mathrm{PQCD}$ behavior is not yet reached even at sizable 

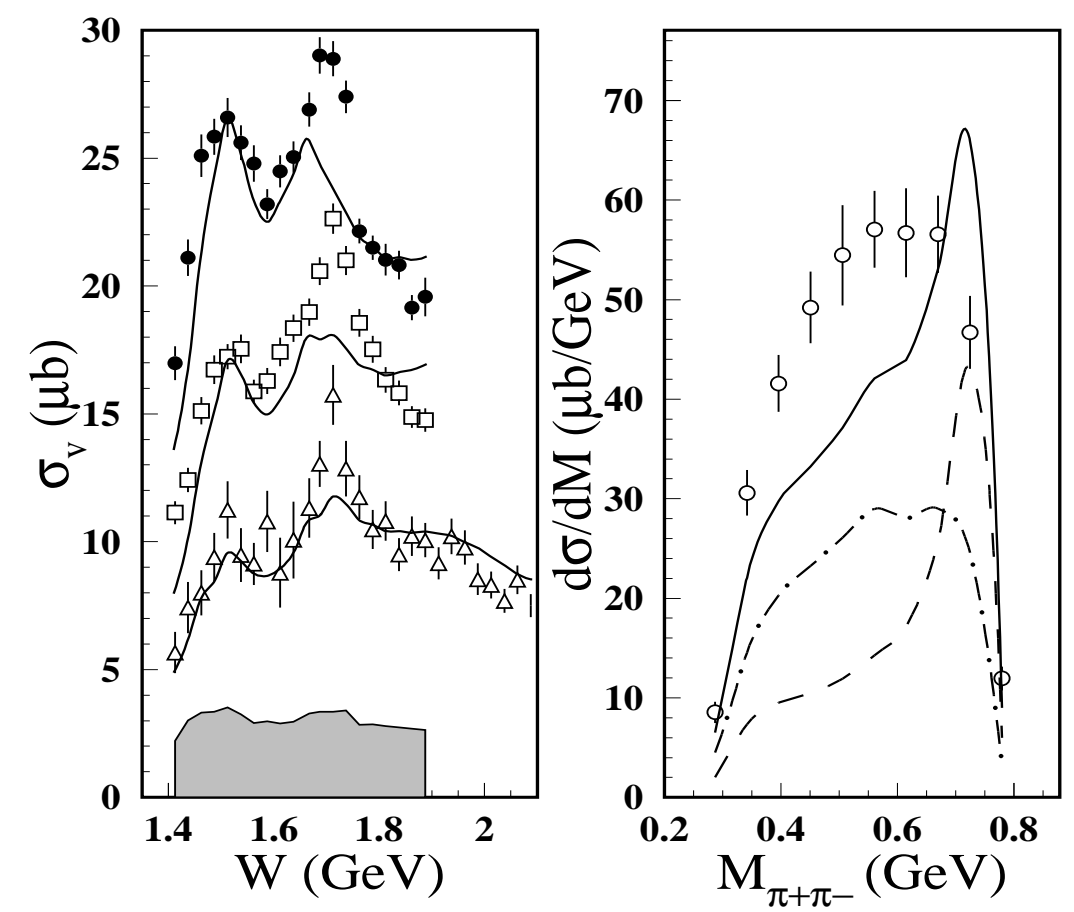

Fig. 2: Left: Total cross section for $\gamma^{*} p \rightarrow p \pi^{+} \pi^{-}$as a function of $\mathrm{W}$ at $<Q^{2}>\sim 0.65 \mathrm{GeV}^{2}$ (full points), $<Q^{2}>\sim 0.95 \mathrm{GeV}^{2}$ (open squares) and $<Q^{2}>\sim 1.3 \mathrm{GeV}^{2}$ (open triangles). Right: $d \sigma / d M_{\pi^{+} \pi^{-}}$at $<Q^{2}>\sim$ $0.95 \mathrm{GeV}^{2}$. The curves represent a phenomenological model. Dashed line includes all resonances, dot-dashed non resonant part while solid lines are the full calculation.

Magnetic Dipole Form Factor

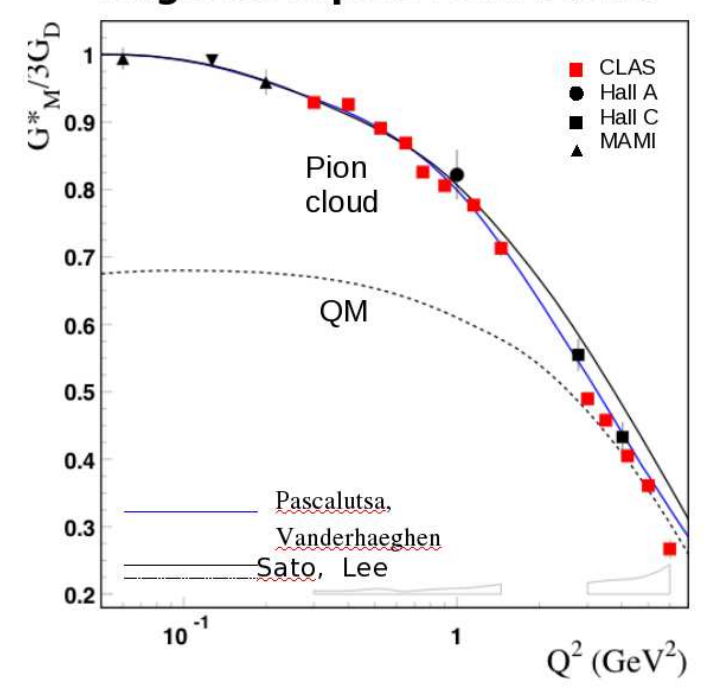

Quadrupole Ratios

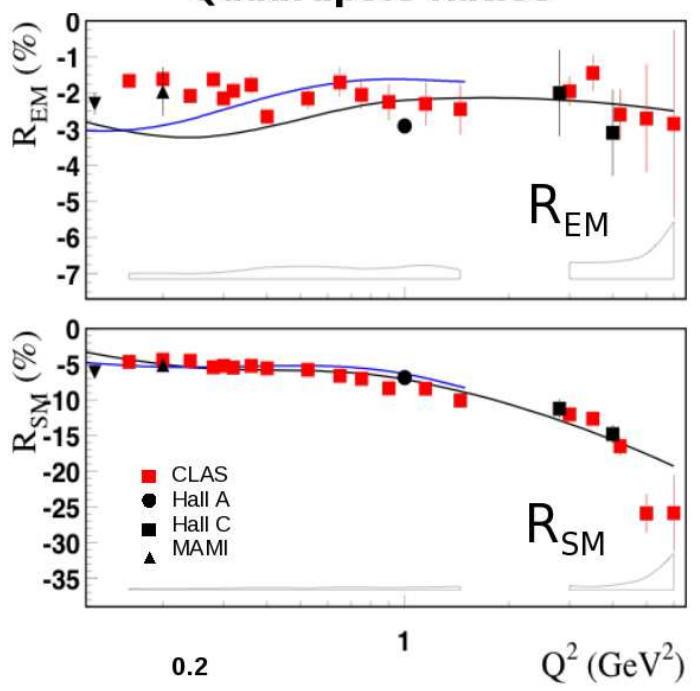

Fig. 3: Results for $Q^{2}$ dependence of the electric $\left(E_{1}\right)$ and scalar $\left(S_{1}\right)$ quadrupole/dipole ratios in $\Delta(1232)$ resonance excitation. Data from other labs as well as various theoretical predictions are also reported.

$Q^{2}$. The ratio $R_{S M}$ also remains negative decreasing with $Q^{2}$. Comparison with microscopic models (relativized quark models, chiral quark soliton model and dynamical models) shows that the simultaneous description of both $R_{E M}$ and $R_{S M}$ is achieved when the pion cloud is add to the usual constituent quark degrees of freedom. 


\section{Meson spectroscopy: the present and the future}

Meson spectroscopy has some advantages in looking for exotic configurations: the $q \bar{q}$ system is easier to study than the baryon spectrum and theoretical arguments support the idea that gluonic degrees of freedom should be accessible studying the meson spectrum and identifying exotic configurations in hybrid mesons. Lattice QCD calculations suggest that a linear potential between quarks is behind the confinement of hadrons. In a particular model, self interacting gluons form a string-like flux tube between the interacting pair: while normal mesons have quantum numbers compatible with a flux tube in the ground state, in hybrid mesons, gluon degrees of freedom of the excited flux tube add explicitly to the quark quantum numbers resulting in exotic combination of total angular momentum, parity and C-parity. The identification of states with particular $J^{P C}$ combinations, as $0^{--}, 0^{+-}, 1^{-+}, 2^{+-} \ldots$ is an unambiguous experimental signature for the presence of gluonic degrees of freedom in the spectrum of mesonic states. The flux tube excitation will be induced by using a photon beam. Photoproduction of exotics has many advantages compared to traditional hadro-production (pion or kaon beams): there are some theoretical arguments predicting that exotics are more likely produced by the interaction with a photon, a spin 1 probe, and that the expected production rate should be comparable as for regular mesons. With a $12 \mathrm{GeV}$ electron beam, there are only few choices to produce a high intensity, polarized, tagged photon beam: bremsstrahlung and quasi-real electro production. In the first case, the primary electron beam hitting a thin diamond, produces a coherent bremsstrahlung photon beam of about $10^{7}-10^{8} \mathrm{\gamma} / \mathrm{s}$ with energy ranging between 6 and $11 \mathrm{GeV}$ and reasonable degree of linear polarization $(\sim 40 \%)$. This is a widely used technique used extensively to study baryon spectroscopy in the Hall-B with the existing $6 \mathrm{GeV}$ primary electron beam. The JLab-12 new dedicated experimental hall (Hall-D) will adopt this technique to study meson spectroscopy. The other option to produce a photon beam with above characteristics, is the so-called quasi-real electro-production. It consists in performing a standard electro-scattering on the target at very low photon virtuality $\left(Q^{2}<10^{-3}\right)$ so that the virtual photon can be assimilated to a real photon. In this kinematic a high flux of linear polarized quasi-real photons, comparable to what obtained by using the bremmstrahlung, is easily produced. Detection of the low energy $(\sim 1-3 \mathrm{GeV})$ scattered electron at very small angle, about $1^{\circ}$, allows one to tag the quasi-real photon four momentum. This technique was used in the past to produce high energy $(\sim 100 \mathrm{GeV})$ photon beam at CERN (Omega Collaboration) and DESY (ZEUS experiment). At our knowledge no attempts were made with a $10-\mathrm{GeV}$ electron beam. Some tests were performed in the actual configuration of the CLAS experiments with the $6 \mathrm{GeV}$ electron beam, by looking for hadronic events with no electron detected in the CLAS calorimeters acceptance $\left(>5^{\circ}\right)$, and final state compatible with the assumption of a forward-going electron. The reconstructed mass spectrum of $\pi^{0} \pi^{0}$ and $\pi^{0} \eta$ show clear evidence of rare mesons expected in this channels $\left(f_{0}(980), f_{2}(1270), a_{0}(980)\right)$ demonstrating that this technique works quite well in the present and future kinematic conditions of Jefferson Lab. The implementation of a quasi-real photon beam in the Hall-B at JLab-12 is currently under study. The two complementary techniques to produce the photon beam and the different experimental equipments of the two halls, the spectrometer CLAS12 in Hall-B based on a toroidal magnetic field and the GLUEX solenoidal spectrometer in Hall-D, will allow to explore the whole kinematic of meson photoproduction in the best experimental condition and to perform independent checks of possible findings. The study of meson spectrum already started by using data collected in CLAS and first progresses in developing the analysis tools (partial wave analysis) necessary to identify exotic mesons is being doing both on well and poorly known meson states, such as the $\rho$ and the $f_{0}(980)$ respectively [4]. As an example, Fig. 4 shows the results of a partial wave analysis of channel $\gamma p \rightarrow p \pi^{+} \pi^{-}$. On the upper panel the prominent peak of the $\rho$-meson dominates the $\pi-\pi P$-wave differential cross section. In the lower panel, the $S$-wave, shows a clear variation in the vicinity of the $f_{0}(980)$. It has to be noted that this is the first time that the $f_{0}(980)$ meson has been measured in a photoproduction experiment. The evidence of the $f_{0}(980)$ signal in the $S$-wave is a sign that photoproduction may indeed be a good tool for accessing meson resonances other than vector meson states. 
Other experiments using both the standard bremsstrahlung photon beam, up to $5 \mathrm{GeV}$, and the new proposed technique has just been accomplished or are scheduled for the next year. We expect to have physics results, as well as a test of the whole analysis procedure in the near future.
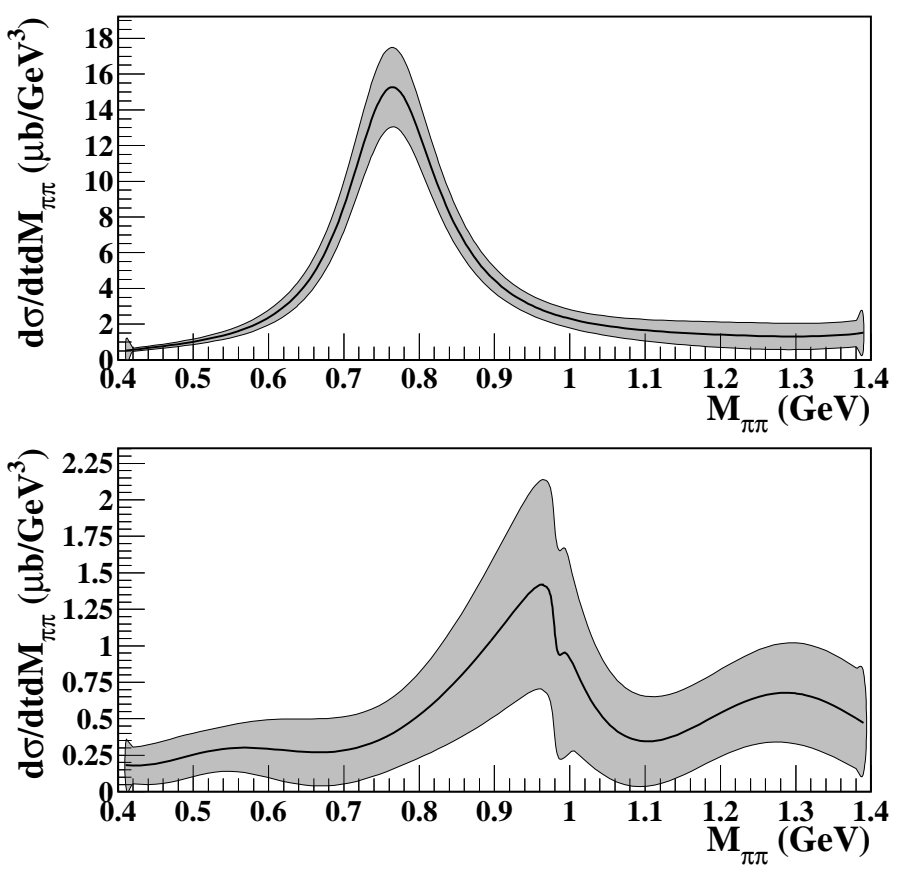

Fig. 4: Partial wave cross sections $d \sigma / d t d M_{\pi \pi}$ for the reaction $\gamma p \rightarrow p \pi^{+} \pi^{-}$in the photon energy bin $3.2<$ $E_{\gamma}<3.4 \mathrm{GeV}$ and momentum transfer $0.5<-t<0.6 \mathrm{GeV}^{2}$. The top and bottom panels show the $P$ - and the $S$-wave, respectively.

\section{Conclusions}

Electromagnetic reactions provide insight into the world of hadronic structure, ranging from different aspects in the excitation of nucleon resonances to the partonic picture of hard processes. The large and broad experimental effort of the CLAS collaboration at Jefferson Laboratory is providing a wealth of new data that will help to clarify our understanding of nucleon structure and nuclear dynamics in the intermediate energy region, which is the domain of non-perturbative QCD. The new data put stronger constraints on QCD-inspired calculations showing that this kinematical region can be described as a non perturbative partonic regime, where microscopic degrees of freedom (gluons and quarks) are combined with a low-energy picture of hadrons (constituent quarks, dressed gluons).

\section{References}

[1] B. Mecking et al., Nucl. Instrum. and Meth. A503, 513 (2003).

[2] M. Ripani et al., Phys. Rev. Lett. 91, 022002 (2003).

[3] M. Ungaro et al. (CLAS Collaboration), Phys. Rev. Lett.97, 112003 (2006).

[4] M. Battaglieri et al. (CLAS Collaboration), Phys. Rev. Lett. 102, 102001 (2009). 